\title{
Phase shift and spectral function from PWA
}

\author{
Pok Man $\mathrm{Lo}^{1, *}$ \\ ${ }^{1}$ University of Wroclaw, Wroclaw, Poland
}

\begin{abstract}
I illustrate a robust method for extracting an effective phase shift and an effective spectral weight for a coupled-channel system. These quantities are useful for describing the thermodynamics of an interacting hadron gas within the S-matrix formulation.
\end{abstract}

\section{S-matrix formulation of statistical mechanics}

The S-matrix formulation of statistical mechanics by Dashen, Ma, and Bernstein [1] expresses the grand canonical potential in terms of the scattering matrix elements. When applied to describe the system of interacting hadrons [2], the logarithm of the partition function can be written as a sum of two parts:

$$
\ln Z=\ln Z_{0}+\Delta \ln Z
$$

where

$$
\ln Z_{0}=V \times \sum_{i \in \mathrm{gs}} d_{i} \int \frac{d^{3} k}{(2 \pi)^{3}} e^{-\beta \sqrt{k^{2}+m_{i}^{2}}}
$$

is the grand potential for an uncorrelated gas of particles that do not decay under the strong interaction (i.e. ground-state particles), such as pions, kaons, and nucleons. The interacting part of the grand potential, $\Delta \ln Z$, can be written in the form

$$
\Delta \ln Z=V \times \int d \sqrt{s} \frac{d^{3} P}{(2 \pi)^{3}} e^{-\beta \sqrt{P^{2}+s}} \rho_{\mathrm{eff}}(\sqrt{s})
$$

where $\sqrt{s}$ is the invariant mass of the relevant scattering system. The quantity $\rho_{\text {eff }}(\sqrt{s})$ can be understood as an effective level density due to the interaction. A key step of the formulation is to relate $\rho_{\text {eff }}(\sqrt{s})$, in the low density limit where only binary collisions are important, to the (derivative of) scattering phase shifts

$$
\rho_{\mathrm{eff}}(\sqrt{s}) \rightarrow \rho_{\mathrm{smat}}(\sqrt{s})=\sum_{\mathrm{int}} d_{I J} \times \frac{d}{d \sqrt{s}}\left(\frac{1}{\pi} \delta_{I J}(\sqrt{s})\right) .
$$

\footnotetext{
*e-mail: pmlo@ift.uni.wroc.pl
} 

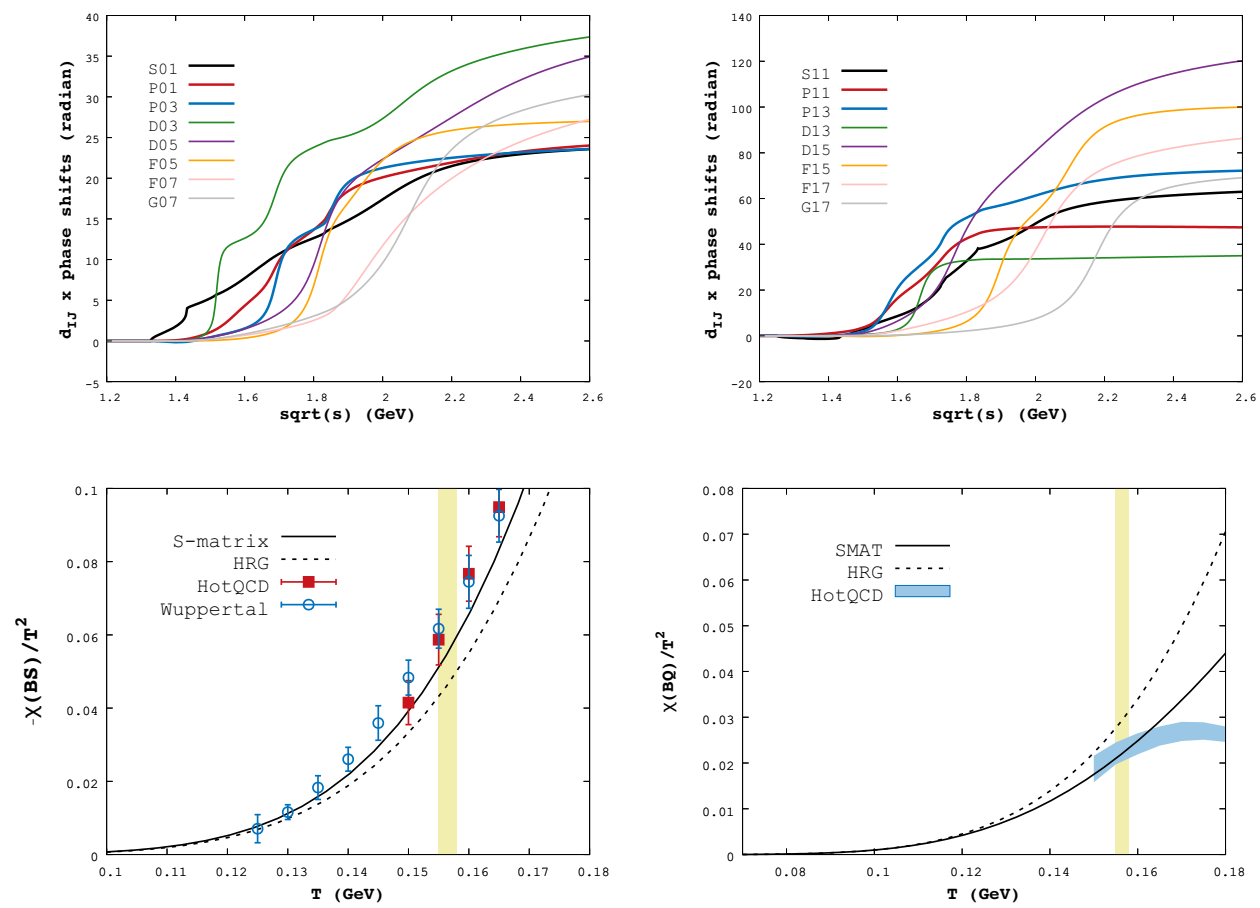

Figure 1. Top: The generalized phase shift function $Q^{l}(s)$ extracted from the coupled-channel PWA in Ref. [5]. Shown in the figures are the major channels contributing to the thermodynamics. Bottom: Fluctuation observables computed using the empirical phase shifts, compared with those obtained from the HRG model and LQCD.

Here the sum is over all interaction channels, $d_{g}$ is the relevant degeneracy factor, and $\delta_{I J}(\sqrt{s})$ is the scattering phase shift. The corresponding results from the standard Hadron Resonance Gas (HRG) model can also be expressed in this form, with the replacement

$$
\rho_{\mathrm{eff}}(\sqrt{s}) \rightarrow \rho_{\mathrm{HRG}}(\sqrt{s})=\sum_{\mathrm{res}} d_{I J} \times \frac{d}{d \sqrt{s}}\left(\theta\left(\sqrt{s}-m_{\mathrm{res}}\right)\right),
$$

where the sum is now over all resonances, treated as point-like particles.

The formalism can be extended to describe a coupled-channel system. In Refs. [3, 4], the following recipe is suggested to extract an effective phase shift function $Q(\sqrt{s})$

$$
\begin{aligned}
Q(\sqrt{s}) & \equiv \frac{1}{2} \operatorname{Im}(\operatorname{tr} \ln \mathrm{S}) \\
& =\frac{1}{2} \operatorname{Im}(\ln \operatorname{det} \mathrm{S}) .
\end{aligned}
$$

This formula has been applied to extract an effective phase shift for the $S=-1$ hyperon system, based on a coupled-channel PWA study [5] by the Joint Physics Analysis Center (JPAC) collaboration. In this case the (complex) determinant of a $(16 \times 16) \mathrm{S}$-matrix is numerically 
computed at each CM energy $\sqrt{s}$, for each partial wave. The phase shifts obtained are shown in Fig. 1.

Using these phase shifts one can calculate the various thermal observables. An interesting quantity, studied also by the Lattice QCD [6,7], is the second order baryon strangeness correlation, $\chi_{B S}$. The result is shown in Fig. 1. Also shown is the result from a similar study [8] on the observable $\chi_{B Q}$.

Computing the thermal observables based on these empirical phase shifts allow the proper treatment of resonances, and the natural incorporation of additional hyperon states which are not listed in the Particle Data Group. This leads to an improved description of the lattice data over the standard HRG model, for both the charge baryons and the $S=-1$ hyperons.

This research benefits greatly from the discussion with Hans Feldmeier and Bengt Friman in GSI. Support from the Extreme Matter Institute EMMI at the GSI is gratefully acknowledged. This work was partially supported by the Polish National Science Center (NCN), under Maestro grant DEC2013/10/A/ST2/00106.

\section{References}

[1] R. Dashen, S. K. Ma and H. J. Bernstein, Phys. Rev. 187, 345 (1969)

[2] R. Venugopalan and M. Prakash, Nucl. Phys. A 546, 718 (1992)

[3] P. M. Lo, Eur. Phys. J. C 77, 533 (2017)

[4] C. Fernandez-Ramirez, P. M. Lo, and P. Petreczky, arXiv:1806.02177 [hep-ph]

[5] C. Fernandez-Ramirez, I. V. Danilkin, D. M. Manley, V. Mathieu and A. P. Szczepaniak, Phys. Rev. D 93, 034029 (2016)

[6] A. Bazavov et al., Phys. Rev. Lett. 113, 072001 (2014)

[7] S. Borsanyi, Z. Fodor, S. D. Katz, S. Krieg, C. Ratti and K. Szabo, J. High Energy Phys. 1201, 138 (2012)

[8] P. M. Lo, B. Friman, K. Redlich and C. Sasaki, Phys. Lett. B 778, 454 (2018) 